\title{
Scar Pregnancy: A Case Series Involving Two Medical College Hospitals in West Bengal
}

\author{
Indranil Dutta ${ }^{1}$, Abhijit Haldar ${ }^{2}$, Mainak Nath ${ }^{3}$
}

\begin{abstract}
Scar pregnancy is increasing day by day due to the increasing number of cesarean section as well as operative intervention on uterus. Though there are improved diagnostic modalities in recent era, there is certain a deficiency of knowledge of specific diagnosis and treatment among the obstetricians and radiologists. Only few case reports are available online, but there are more cases of scar pregnancy which are underdiagnosed leading to high morbidity and even mortality. In this case series, we report 11 cases of scar ectopic pregnancy with different presenting features and treatment. Though in our case series there is no mortality, morbidity is seen to be high. We tried to increase the suspicion of scar ectopic pregnancy among obstetricians and radiologists to reduce these morbidities, especially in the cases of previous cesarean section and previous myomectomies. Keywords: Cesarean section, Dilation and evacuation, Ectopic, Myomectomy, Scar ectopic pregnancy, Ultrasonography.

Journal of South Asian Federation of Obstetrics and Gynaecology (2020): 10.5005/jp-journals-10006-1756
\end{abstract}

\section{INTRODUCTION}

Scar pregnancy is defined as an ectopic pregnancy situated in the myometrium of a previous cesarean scar. ${ }^{1}$ This rise is due to more number of operative interventions, especially cesarean section. Scar ectopic pregnancy following cesarean section is the rarest form of ectopic pregnancy wherein the gestational sac implants into the fibrous tissue scar of a previous cesarean section. It accounts for approximately $6.1 \%$ of ectopic pregnancies and $0.14 \%$ in previous section cases. ${ }^{2,3}$

Not only the scar of cesarean section, but even uterine scar due to myomectomy or perforation during the dilation and evacuation (D\&E) procedures may also be a site for implantation leading to scar pregnancy. Intramyometrial ectopic pregnancy is one of the rarest types of ectopic pregnancy constitutes less than $1 \%$ of the total number and is the rarest type. ${ }^{4}$

The lower segment cesarean section (LSCS) delivery is one of the most common routes of operation in today's time. ${ }^{5}$ In many countries around the world, obstetric practice has witnessed an increasing frequency in cesarean deliveries which has increased from 21.8 to $25.4 \%{ }^{6}$

The main problem is that in case of scar pregnancy, the placenta attaches to previous scar which is already thinned out leading to more chances of placenta accreta/increta/percreta involving bladder sometimes. Generally, this placental attachment leads to thinning of scar which eventually gives up as the pregnancy progresses leading to life-threatening complications.

So early diagnosis and specific treatment approaches are necessary to avoid the complications caused by scar pregnancy.

\section{Case Description}

\section{Case 1}

A patient aged 32 years, G2P1L1, with cesarean section done 5 years back had come to the emergency room (ER) of IQCMC with heavy bleeding through the vagina with the products of conception hanging through the cervical OS.

The patient had taken MTP pill without prescription. The patient was taken up for emergency D\&E as the vitals was continuously
'Department of Obstetrics and Gynaecology, IQ City Medical College Durgapur, West Bengal, India

${ }^{2,3}$ Department of Obstetrics and Gynaecology, College of Medicine and JNM Hospital, West Bengal University of Health Sciences, Kalyani, West Bengal, India

Corresponding Author: Indranil Dutta, Department of Obstetrics and Gynaecology, Institute of IQ City Medical College, Durgapur, West Bengal, India, Phone: +91 9051556424, e-mail: drindranildutta@gmail. com

How to cite this article: Dutta I, Haldar A, Nath M. Scar Pregnancy: A Case Series Involving Two Medical College Hospitals in West Bengal. J South Asian Feder Obst Gynae 2020;12(1):51-58.

Source of support: IQ City Medical College, COM JNM Hospital

Conflict of interest: None

deteriorating. As soon as the product was removed from cervical os, torrential bleeding started from some sinuses in anterior wall of the lower segment.

Immediate decision of laparotomy was taken. It was found that there was a scar pregnancy in the previous scar area along with placenta percreta involving bladder (Fig. 1). Finally, the decision for hysterectomy was taken and the specimen was removed (Fig. 2). The patient was doing well postoperatively. Histopathology (HPE) showed the following diagnosis- "section from ruptured uterine wall shows decidualization, hemorrhage, and chorionic villi with trophoblastic cell infiltration in myometrium. Features are consistent with scar pregnancy."

\section{Case 2}

A 25-year-old woman, G2P1L1, previous 1 CS, 9-week history of amenorrhea, presented to the ER of COM JNMH with acute lower abdominal pain and spotting with features of severe pallor, low BP, tachycardia. She had taken MTP kit by herself 2 weeks prior to the presentation which caused no sufficient bleeding. For this, she visited a gynecologist outside and ultrasonography (USG) was done at a local center. The USG reported as an incomplete 


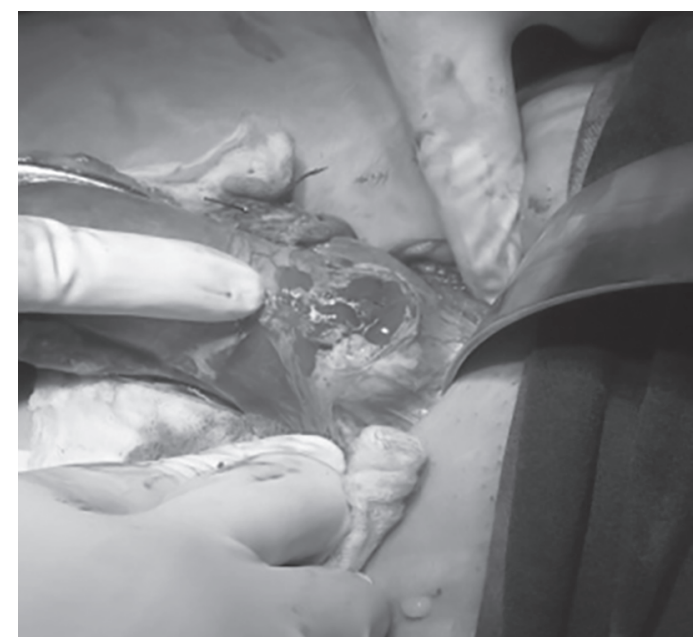

Fig. 1: Intraoperative picture of uterus showing scar ectopic pregnancy area (Case 1)

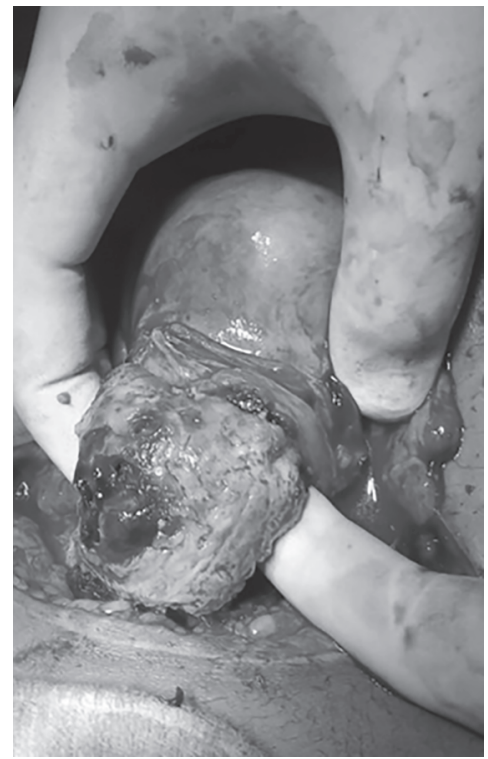

Fig. 3: Intraoperative picture of uterus showing scar ectopic pregnancy area (Case 2)

abortion. The departmental USG after admission by obstetrician at our medical college (COM JNMH) revealed hemoperitoneum with a complex cystic structure of at lower part of the uterus. Immediate laparotomy was done. It was found to be a scar ectopic pregnancy which was bleeding after rupture. Scar pregnancy was confirmed by HPE (Figs 3 and 4).

\section{Case 3}

A 19-year-old unmarried girl came to the ER of IQCMC with severe tender lower abdomen, tachycardia $120 \mathrm{~min}, \mathrm{BP} 80 / 60 \mathrm{~mm} \mathrm{Hg}$. We suspected it to be a case of ectopic pregnancy, and urinary pregnancy test (UPT) was positive.

(Past History: She had been operated 2 months back at a reputed medical college hospital in south for myomectomy of fibroid approx. $12 \mathrm{~cm} \times 10 \mathrm{~cm}$ ). She was quickly taken up for laparotomy where it was found that the previous myomectomy scar (Figs 5 and 6) had ruptured with a pregnancy within (Fig. 7). It was not a case of scar rupture which was proved by histopathology,

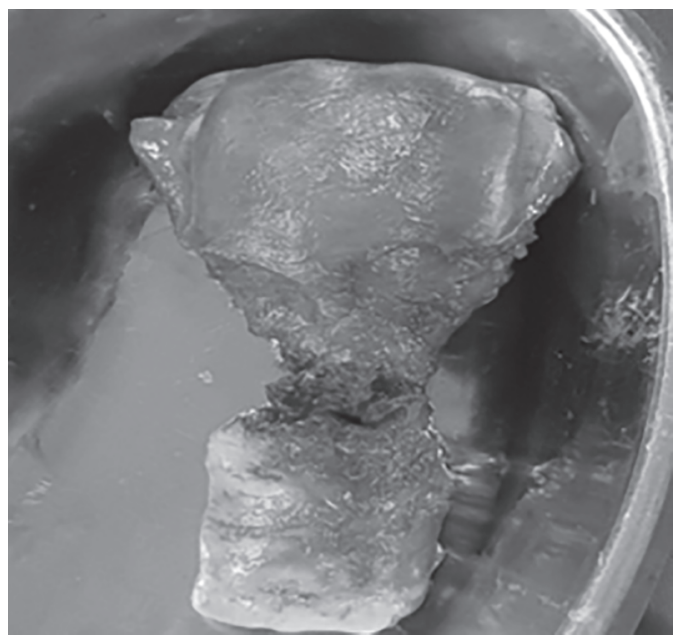

Fig. 2: Post-hysterectomy specimen (Case 1)

but it was reported to be a case of scar ectopic pregnancy. The patient was managed well with repair of myomectomy scar area and restoration of the anatomy (Figs 5 to 8 ).

\section{Case 4}

A 40-year-old patient G5P2A1L1, previous 2 LSCS, attended ER with complaint of on and off bleeding through the vagina after two unsuccessful attempt of D\&E at two different private hospitals 35 and 15 days prior to the presentation for incomplete abortion. She had a history of hemorrhagic shock in each attempt of D\&E. The first D\&E was done at 8 weeks of amenorrhea. After the second attempt, she had an USG done outside, which reported as fibroid in the anterior lower uterine segment but endometrial cavity empty. Provisionally diagnosed to be a case of submucosal fibroid surface vessel laceration during each D\&E which may have bled. As her urine for pregnancy test was positive, she was put for laparotomy. During the laparotomy, a globular mass protruding out of lower uterine segment seen with hemorrhagic surface, but no adnexal mass was detected. As family was completed and also because of poor initial judgment, the decision of hysterectomy taken with the mass. HPE reported the lower segment SOL of the hysterectomy specimen (Fig. 9) to be a mass with chorionic villi and blood clots suggestive of scar site pregnancy. The picture of longitudinal section of the uterus (Fig. 10) shows the blood clots with gestational tissue invasion to myometrial layer of the uterus.

\section{Case 5}

A 34-year-old patient G3P1A1L1, previous 1 LSCS, attended ER of IQCMC with complaint of on and off bleeding through vagina after one attempt of D\&E at private hospital nearby. She had H/o postLSCS PPH and re-laparotomy for the same. As she was torrentially bleeding during repeat attempt of D\&E at our hospital, she was put for emergency laparotomy on the same OT table. During the laparotomy, a mass was noted at previous scar site (Fig. 11). The scar was opened and ectopic mass was removed (Fig. 12). The previous scar tissue was excised and removed, and fresh edges were repaired to keep the uterus intact. Histopathology confirmed it to be scar pregnancy sac with ectopic tissue, the myometrial scar area which was excised also showed tissue invasion of mild grade. The patient's subsequent scans showed normal anatomy of uterus. 


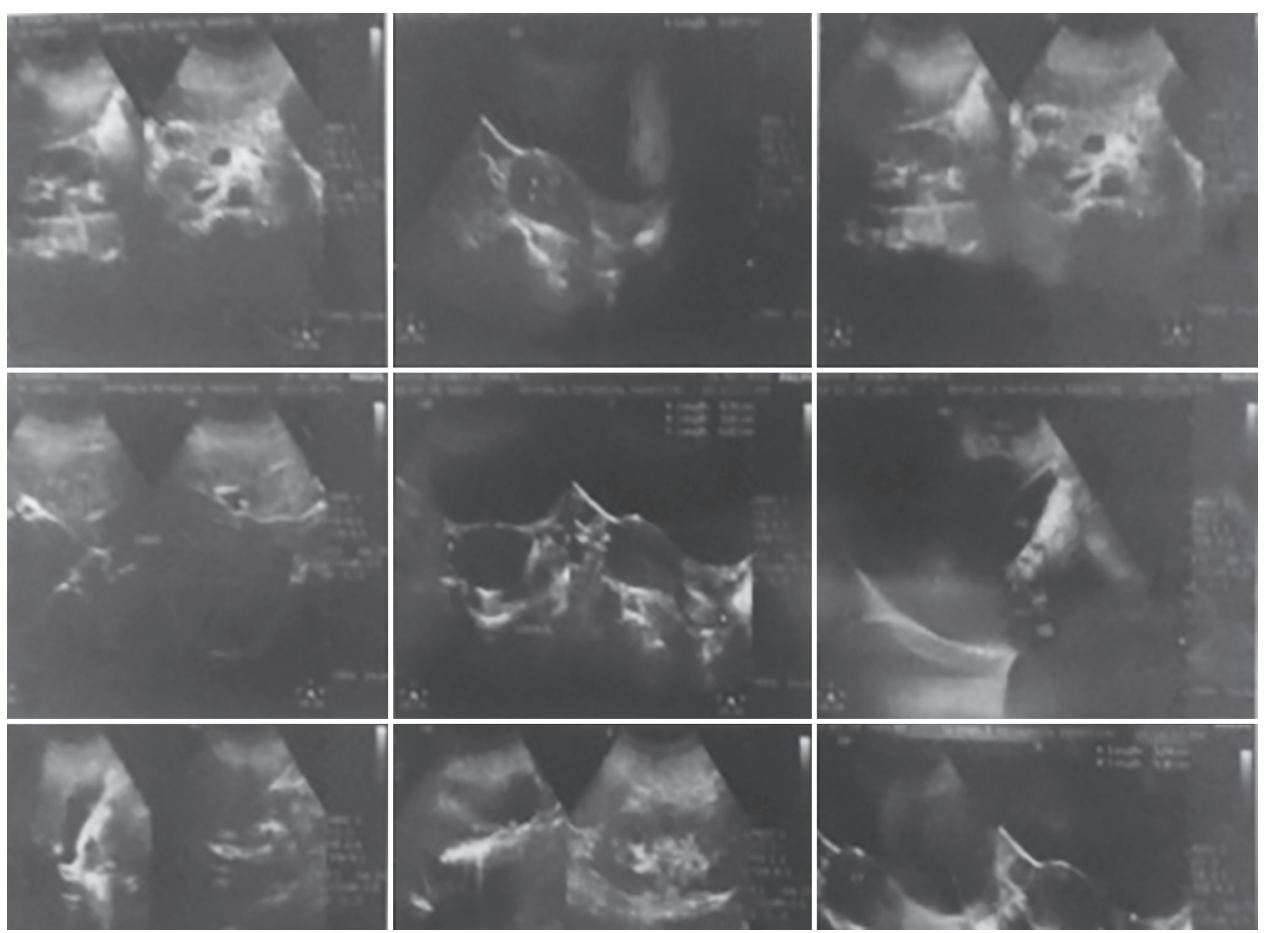

Fig. 4: USG showing scar ectopic pregnancy (Case 2)

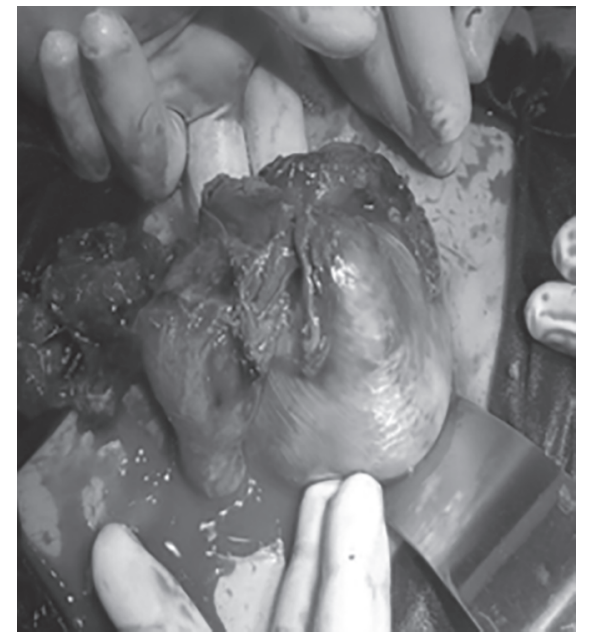

Fig. 5: Intraoperative picture showing previous myomectomy scar area (Case 3)

\section{Case 6}

A 22-year-old patient G2P1A0L1 (previous one CS 1 year back) referred from periphery district hospital with severe bleeding per vagina following D\&E. She had taken MTP kit from a local medical shop with the intention of abortion for 6 weeks of pregnancy without any ultrasound done prior to that. Clinical suspicion and experience led to do an emergency departmental USG which showed a heterogeneous mass $(3 \times 4) \mathrm{cm}$ the lower uterine segment pushing the bladder anteriorly. She was managed by laparotomy and removal of the gestational sac with blood clots from previous scar site (Figs 13 to 15 ).

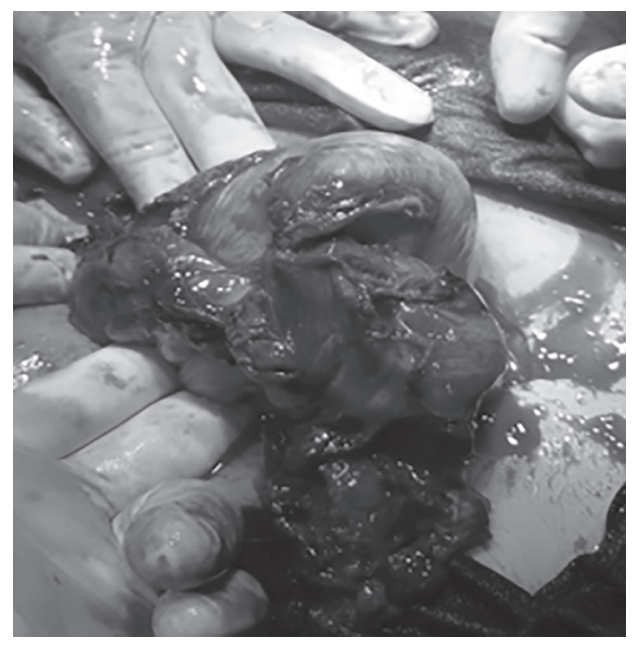

Fig. 6: Intraoperative picture showing previous myomectomy scar area (Case 3)

\section{Case 7}

The patient, G2P1A0L0, presented to ER with severe lower abdominal pain at 8 weeks of amenorrhea. She had lower abdominal tenderness and tachycardia. Emergency ultrasound showed a gestational sac at lower uterine segment at previous CS scar with a live embryo of 7 weeks 2 days by crown-rump length. There was a loss of endomyometrial junction at bladder area-suggestive of impending rupture (Fig. 16). Immediate laparotomy was done, and it was found that the gestational sac with live embryo in it protruding out through the scar area. The sac carefully dissected out with the forming placenta. The scar was 


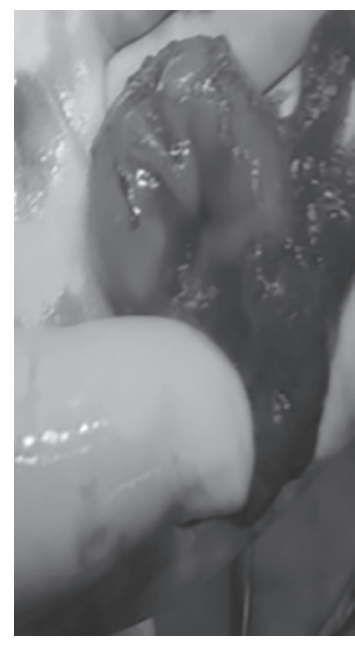

Fig. 7: Ectopic pregnancy sac (Case 3)

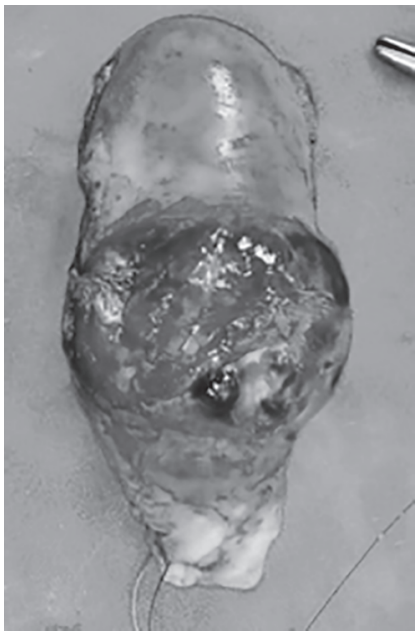

Fig. 9: Post-hysterectomy specimen showing scar ectopic pregnancy (Case 4)

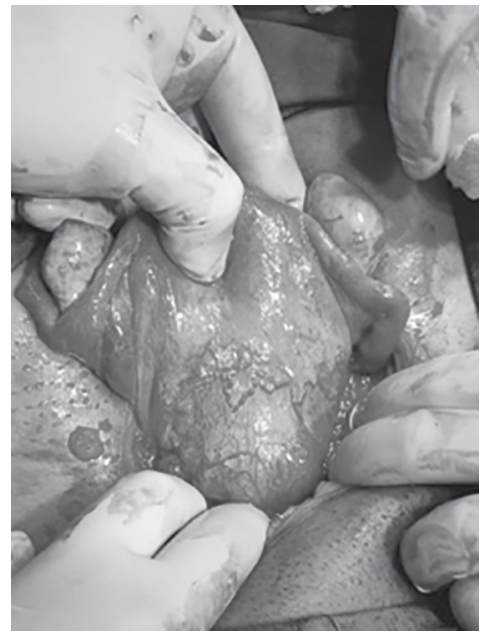

Fig. 11: Intraoperative picture showing scar ectopic pregnancy implantation in lower segment (Case 5)

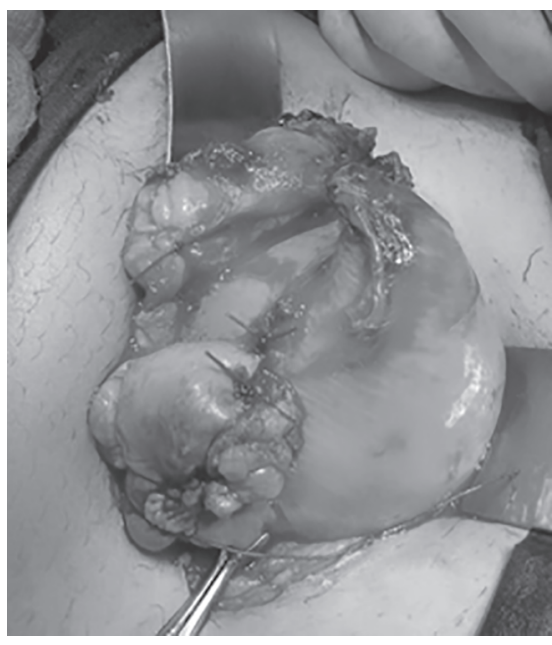

Fig. 8: Post-repair picture (Case 3)

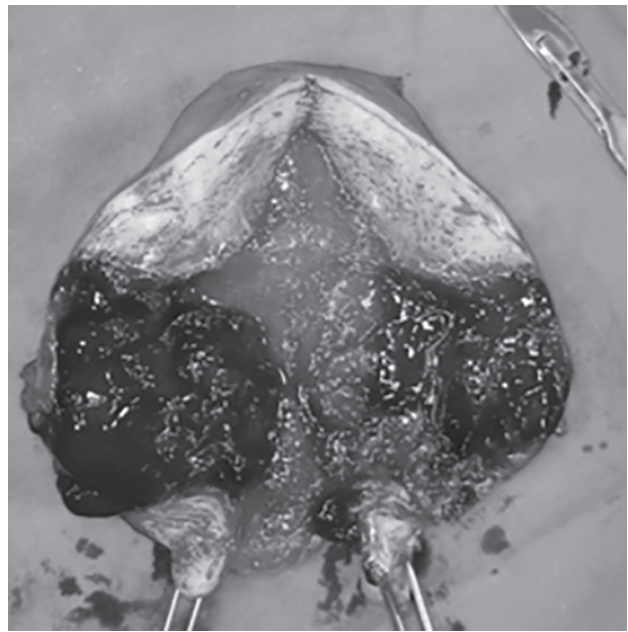

Fig. 10: Cut section of hysterectomy specimen showing involved area (Case 4)

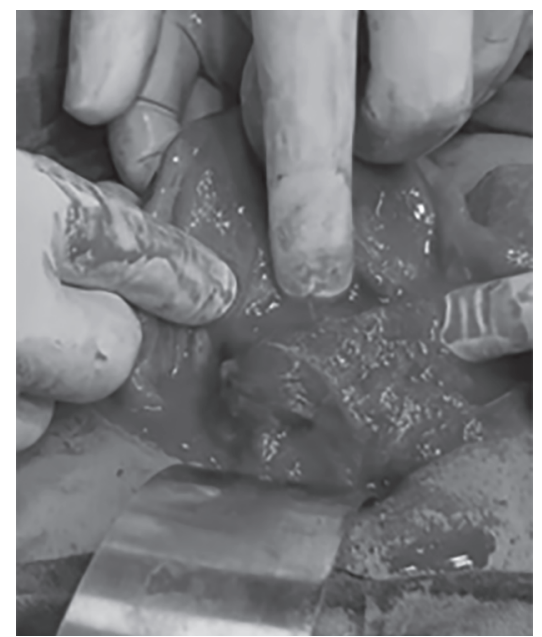

Fig. 12: Post-evacuation picture of lower segment (Case 5) 


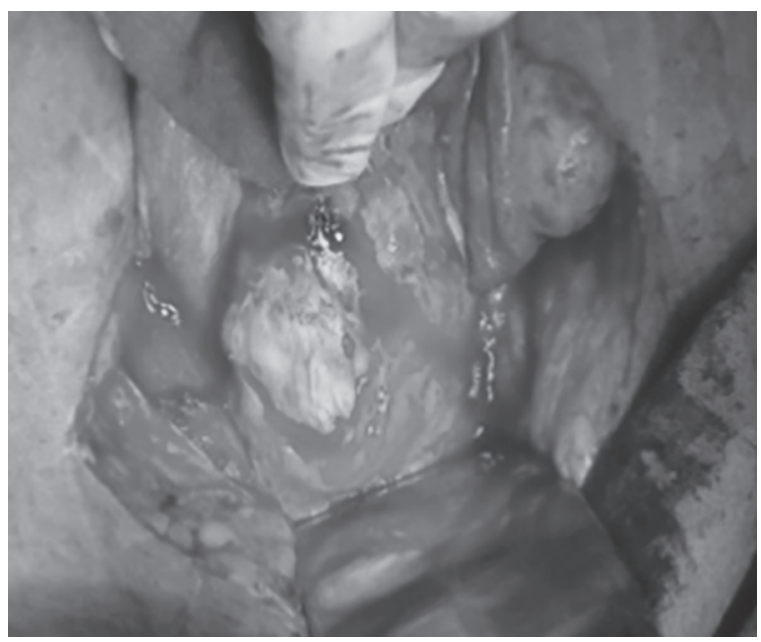

Fig. 13: Intraoperative picture showing scar ectopic pregnancy (Case 6)

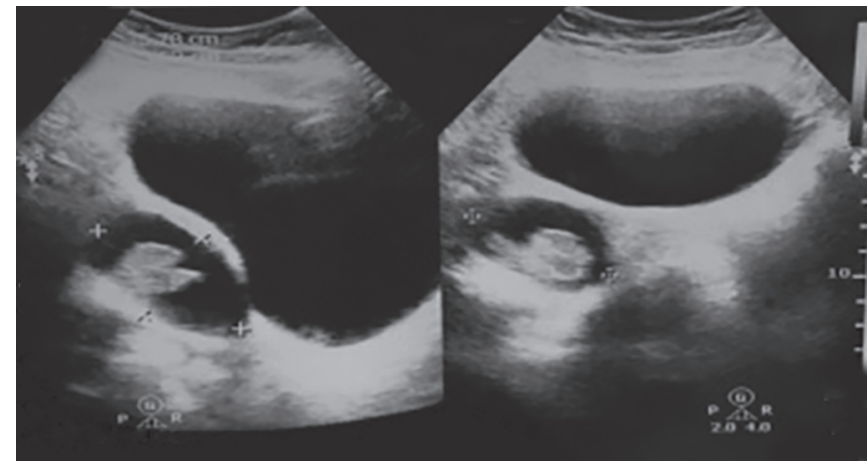

Fig. 15: Ultrasound picture showing scar ectopic pregnancy (Case 6)

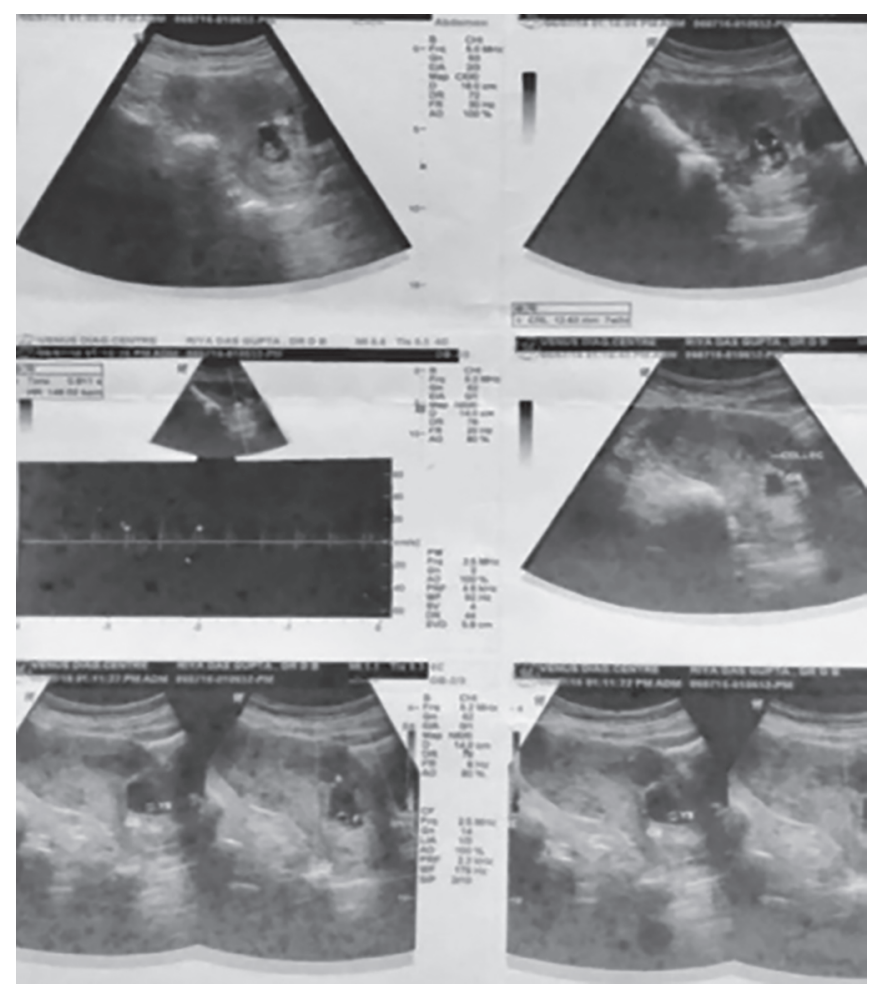

Fig. 17: USG showing gestational sac implanted within previous cesarean scar (Case 8)

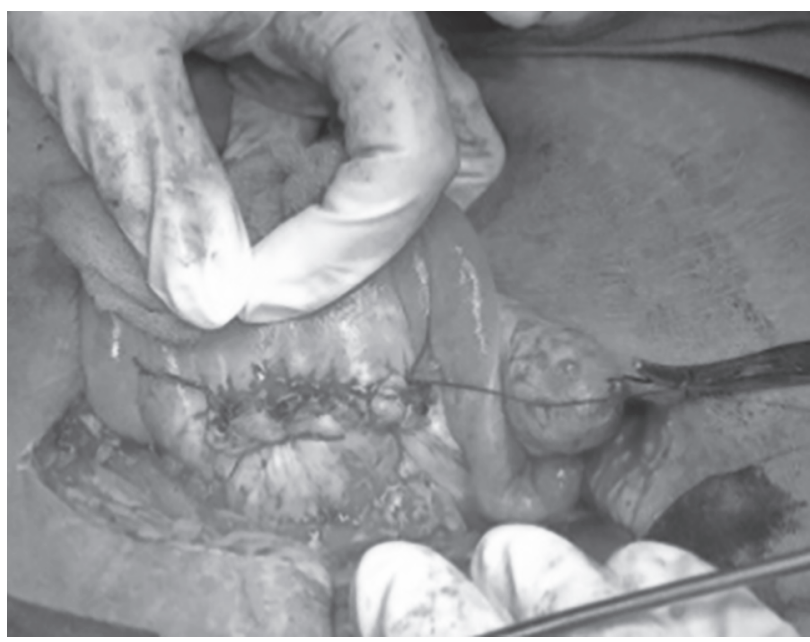

Fig. 14: Intraoperative picture showing scar area post repair (Case 6)

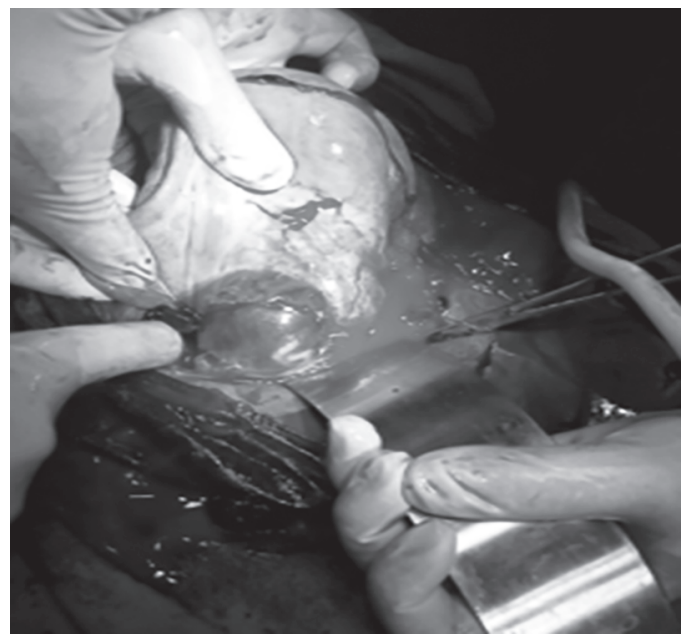

Fig. 16: Intraoperative picture showing uterine scar with gestational sac with live embryo in it protruding out through the scar area (Case 7)

repaired and the specimen was sent for HPE. Postoperative period was uneventful.

\section{Case 8}

A 32-year-old woman, G5P2A2L2, previous 2 LSCS, attended outdoor with a history of one-and-half-month of amenorrhea and sonography with color Doppler suggested gestational sac with embryo of CRL $7.2 \mathrm{~mm}$, implanted within the previous cesarean scar (clearly visible on plate) though reported as gestational sac in endometrial cavity by the radiologist outside. She had a history of failed attempt for termination of pregnancy by taking two consecutive MTP kits. D\&E was done at our hospital depending upon the report only. During the D\&E, very scanty product of conception came out, which raised the suspicion for rechecking of the report with USG plates (Fig. 17). Repeat USG showed gestational sac still present at lower anterior wall of uterus at the previous site with hCG titer of $340 \mathrm{IU} / \mathrm{ml}$. She was managed by injection methotrexate $50 \mathrm{mg}$ single dose weekly with serum beta-hCG estimation weekly till normal value was achieved. She became UPT negative after three injections of methotrexate, and on routine follow-up, she was doing well. 


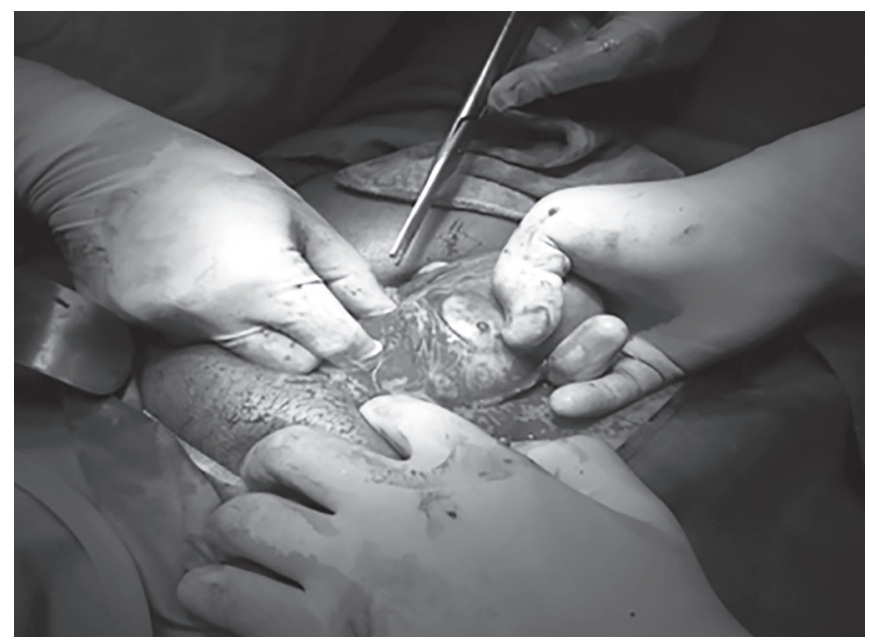

Fig. 18: Intraoperative picture (Case 9)

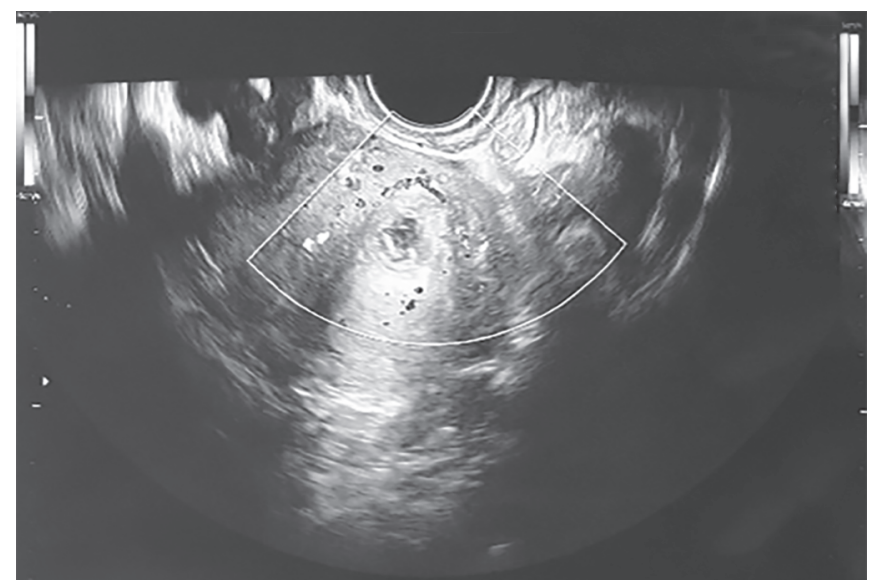

Fig. 20: USG showing myometrial ectopic pregnancy in anterior myometrium (Case 10)

\section{Case 9}

G3P1A1, a 30-year-old woman, attended in the ER with almost same history as Case 6. She also underwent D\&E outside. She also was managed by laparotomy in hospital, but she needed internal iliac artery ligation as her bleeding from the scar repair site could not be controlled. She had extensive adhesion involving bladder, omentum, and CS scar-ectopic pregnancy sac (Figs 18 and 19).

\section{Case 10}

A 23-year-old patient G3P1L1A1 came with H/o bleeding through the vagina for 7 days. She underwent D\&E at a private clinic in Raniganj, West Bengal. Initial USG was done outside which reported it to be a case of anterior wall degenerating fibroid though she was UPT positive with adnexa free and endometrial cavity empty. The USG in our hospital IQCMC reported as a myometrial ectopic/ complex cyst in anterior myometrium wall measuring $1.8 \mathrm{~cm} \times$ $1.7 \mathrm{~cm}$. Serum beta human chorionic gonadotropin (hCG) was done which showed a value of $316 \mathrm{IU} / \mathrm{mL}$. As she had a history of attempted D\&E and suspected perforation by private doctor, repeat D\&E was abandoned as the patient was stable. Scar pregnancy was suspected and put on injection methotrexate 50 mg weekly,

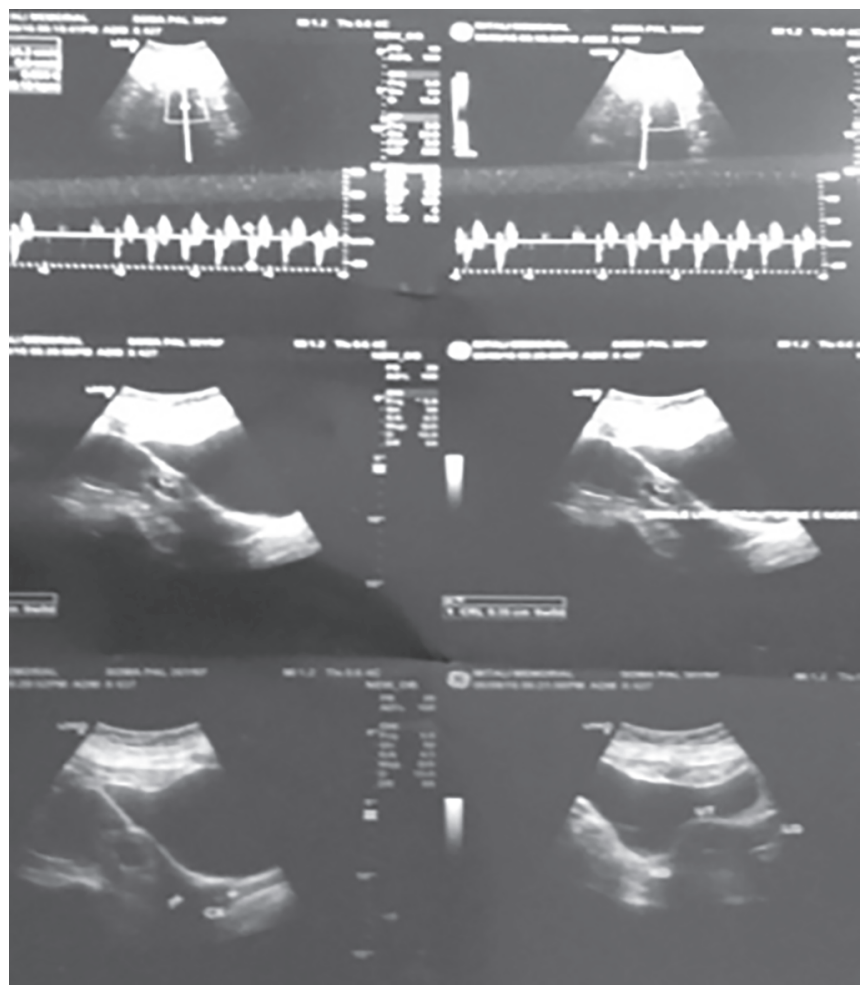

Fig. 19: USG showing scar pregnancy (Case 9)

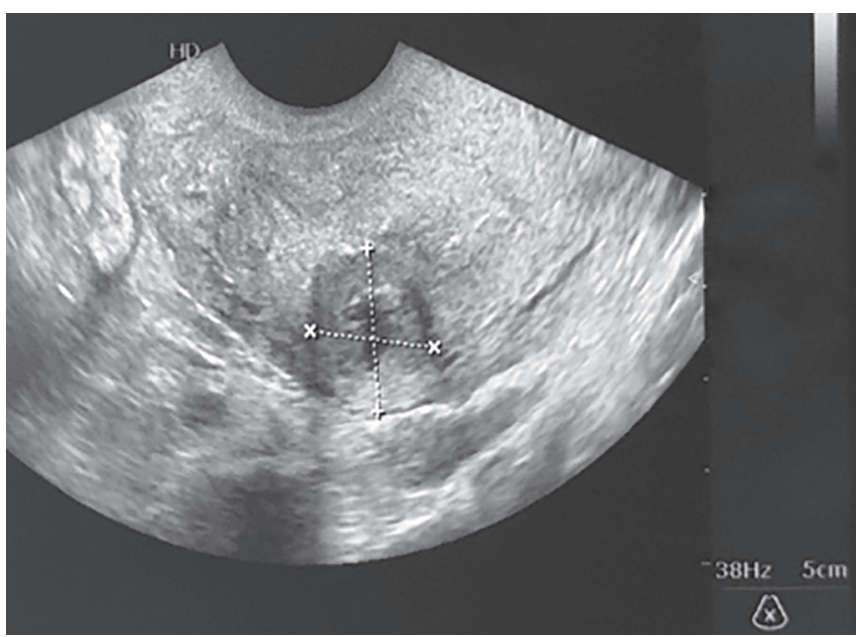

Fig. 21: USG showing myometrial ectopic pregnancy in anterior myometrium (Case 10)

and serial $\beta$-hCG showed satisfactory regression of IQCMC levels. She reached normal $\beta$-hCG level after 4 doses of methotrexate. The patient is doing well (Figs 20 to 22).

\section{Case 11}

A 27-year-old woman, G2P1+0, LUCS 8 years back, LMP 2 months back, UPT +ve, came with bleeding through the vagina from 10 days. The USG showed missed abortion (Fig. 23); hence, 2 tab misoprostol given prior to D\&E operation by a private doctor. She went to Chakdaha SGH with profuse bleeding through the vagina from where she was referred to nearest tertiary center. Hence she came to ER of COM JNM Hospital, Kalyani. The first USG done in COM 


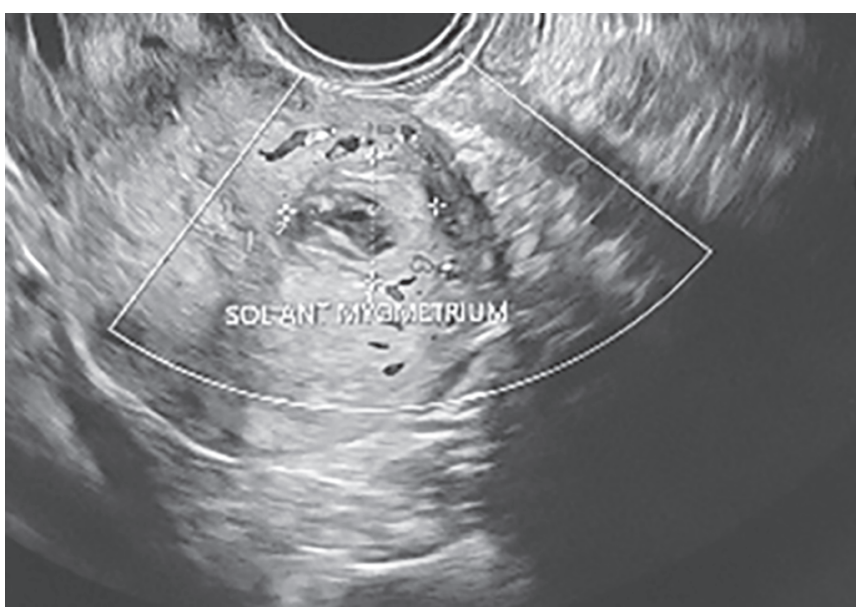

Fig. 22: USG plate picture (Case 10)

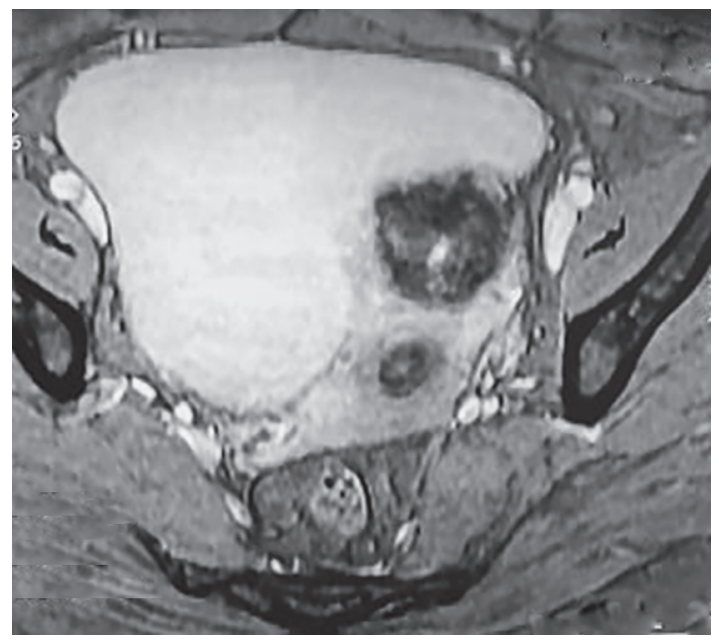

Fig. 24: MRI plates showing scar ectopic pregnancy (Case 11)

JNMH was suggestive of echogenic collection with cystic component within $(8.6 \mathrm{~cm} \times 4.1 \mathrm{~cm})$ in endometrial cavity with diagnosis of partial mole/incomplete abortion; the patient was taken to OT for suction evacuation. During the procedure, torrential bleeding was started and was controlled with uterotonics. The patient was shifted to HDU. The $\beta$-hCG titer of first day showed $95.73 \mathrm{mlU} / \mathrm{mL}$, which goes against suspicion of partial mole; hence, the expert radiologist called for and repeat USG was done which was suggestive of one ill-defined heterogeneous lesion $(52 \times 45) \mathrm{mm}$ seen eccentrically in lower uterine segment, anterior wall at the previous LUCS scar site s/o scar ectopic pregnancy. MRI plates were attached (Fig. 24). The decision was taken for laparotomy after MRI and scar ectopic pregnancy was confirmed which was managed by enucleation of mass and repair of uterus (Figs 25 and 26). The patient is currently doing well.

\section{Discussion}

Scar pregnancy is the rarest type of ectopic pregnancy of which post-cesarean scar is more commonly encountered. As the number of emergency, LUCS has been increased remarkably, and the incidence of cesarean scar ectopic pregnancy has increased dramatically.

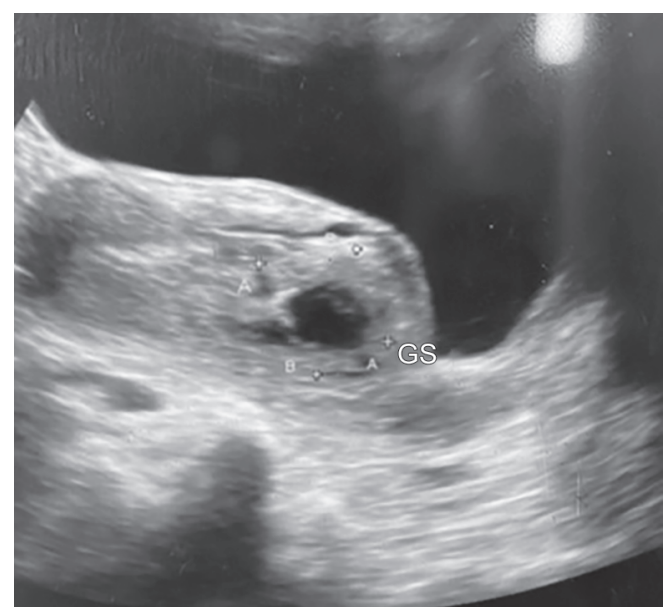

Fig. 23: USG showing previous LUCS scar site with ectopic sac (Case 11)

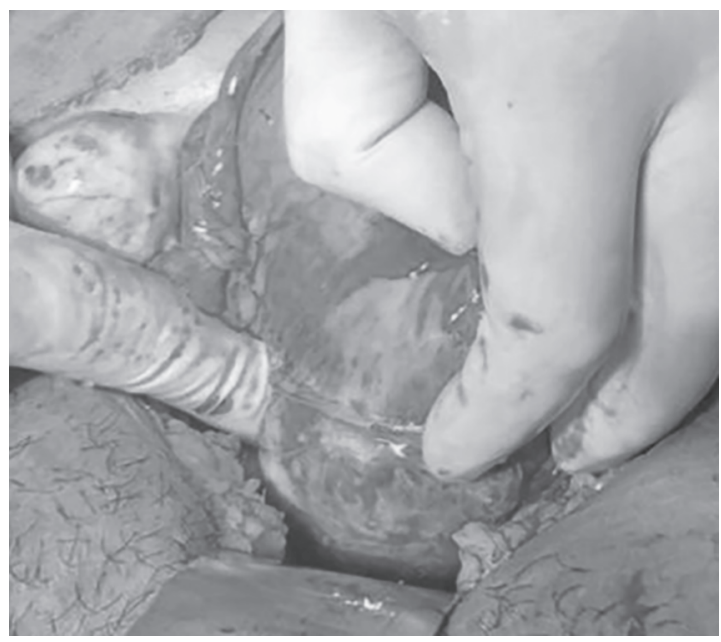

Fig. 25: Intraoperative picture showing scar area (case 11)

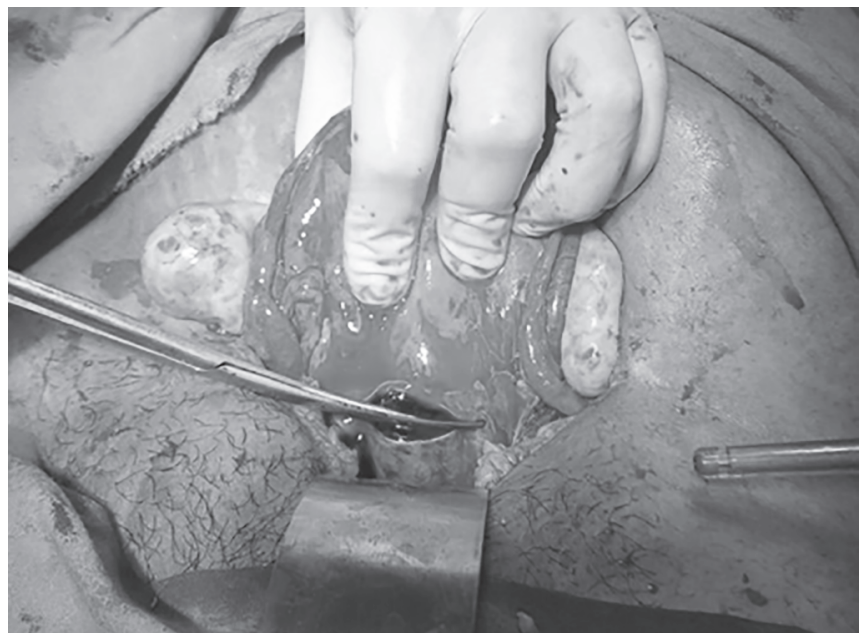

Fig. 26: Intraoperative picture of scar ectopic pregnancy at previous LSCS site (Case 11)

The exact cause of scar pregnancy is still not well understood; it may be due to an existing scar defect or microscopic dehiscent tract 
generated between the prior cesarean scar and the endometrial cavity. ${ }^{7}$

First case of cesarean scar pregnancy was reported in $1978^{8}$ following there are many case reports on cesarean scar ectopic pregnancy which dictates its prevalence and unnatural appearance.

Most of the cases present to emergency with no USG or wrong USG reports which is common in rural areas like ours. High suspicion and knowledge of the radiologist is a necessity for early diagnosis and proper treatment. Even the obstetrician should be well experienced to diagnose a case of scar pregnancy depending on the clinical history and examination.

Most of the cases present with failed attempt of MTP or D\&E with on and off bleeding per vagina. Even a history of hemorrhagic shock during D\&E has been noted in the patients with undiagnosed scar pregnancy (Cases 1, 2, and 5). As MTP kit is easily available in the market, the patients do not hesitate to take it without any USG or any doctor prescription which leads to the emergency presentation at hospital.

Even in cases of myomectomy where the endometrial cavity was breached, a chance of scar ectopic pregnancy at the myomectomy repair site is possible (Case 3 ). If there is a history of perforation during D\&E procedure, a chance of scar ectopic pregnancy should be kept in mind (Case 10).

If early USG is available, operative intervention can be avoided as medical management is a well-established treatment modality to manage early diagnosed scar ectopic pregnancy cases. ${ }^{9,10} \mathrm{We}$ were successful with systemic methotrexate in Cases 8 and 10. Local injection of methotrexate (USG guided and directly into sac) is an established treatment approach. ${ }^{9,10}$

Most of the cases in a peripheral hospital came with the features of shock after attempted D\&E (Cases 4, 5, and 9) when local or systemic methotrexate cannot be a treatment approach. For this reason, chances of laparotomy followed by resection of the ectopic sac or hysterectomy becomes inevitable. Though only resection of the ectopic sac from the scar site and repair of the margins of uterus are successful in most of the cases, many times internal iliac artery ligation or hysterectomy becomes unavoidable to control scar site bleeding. Nowadays, laparoscopic scar pregnancy excision is being practiced by many laparoscopic surgeons, ${ }^{11}$ but in our circumstances where emergency laparoscopic treatment is not available, laparotomy becomes main stay of treatment.

\section{Conclusion}

Early diagnosis by USG and Doppler study is mandatory very useful during routine follow-up but the complication arises when the patient arrives at the emergency with undiagnosed scar pregnancy with associated heavy bleeding. Proper spacing between two pregnancies, proper preconceptional counseling with USG pelvis, and the measurement of previous scar prior to conception are essential followed by early USG during pregnancy to see the site of implantation and invasion into uterine wall.

The obstetrician and the radiologist should be well acquainted with the probability of scar ectopic pregnancy, especially in cases with previous cesarean section and also in the cases of myomectomy. Scar ectopic pregnancy though reported in the cases of cesarean section mostly, in our case series we have found that it can happen in previous myomectomy cases and previous D\&E perforation site.

\section{References}

1. Lai YM, Lee JD. An ectopic pregnancy embedded in the myometrium of a previous caesarean section. Acta Obstet Gynaecol Scand 1995;74(7):573-576. DOI: 10.3109/00016349509024394.

2. Tamada S, Masuyama H, Maki J, et al. Successful pregnancy located in a uterine cesarean scar: a case report. Case Rep Womens Health 2017;14:8-10. DOI: 10.1016/j.crwh.2017.03.003.

3. Seow KM, Huang LW, Lin YH, et al. Cesarean scar pregnancy: issues in management. Ultrasound Obstet Gynecol 2004;23(3):247-253. DOI: 10.1002/uog.974.

4. Ravikanth R. Intramyometrial gestation: a rare localization of ectopic pregnancy. Trop J Obstet Gynaecol 2017;34(1):73-75. DOI: 10.4103/ TJOG.TJOG_2_17.

5. Kambo I, Bedi N, Dhillon BS, et al. A critical appraisal of cesarean section rates at teaching hospital in India. Int J Gynaecol Obstet 2002;79:151-158. DOI: 10.1016/S0020-7292(02)00226-6.

6. Akolekar R, Pandit SN, Rao BS. The cesarean birth FOGSI publications. 1st ed., National Book Depot; 2010. p. 1-3.

7. Godin PA, Bassil S, Donnez J. An ectopic pregnancy developing in a previous caesarean section scar. Fertil Steril 1997;67(2):398-400. DOI: 10.1016/S0015-0282(97)81930-9.

8. Larsen JV, Solomon MH. Pregnancy in a uterine scar sacculusan unusual cause of postabortal haemorrhage. S Afr Med J 1978;53(4):142-143.

9. Feichtinger W, Kemeter P. Conservative treatment of ectopic pregnancy by transvaginal aspiration under sonographic control and methotrexate injection. Lancet 1987;1(8529):381-382. DOI: 10.1016/ S0140-6736(87)91750-8.

10. Yankowitz J, Leake J, Huggins G. Cervical ectopic pregnancy; review of the literature and report of a case treated by single dose methotrexate therapy. Obstetr Gynaecol Surv 1990;45(7):405-414. DOI: 10.1097/00006254-199007000-00001.

11. Pirtea L, Balint O, Secosan C, et al. Laparoscopic resection of cesarean scar ectopic pregnancy after unsuccessful systemic methotrexate treatment. J Minim Invasive Gynecol 2019;26(3):399-400. DOI: 10.1016/j.jmig.2018.06.003, Epub 2018 Jun 8. 